\title{
Avaliação do Controle Interno e Tamanho dos Órgãos sob Jurisdição do Tribunal de Contas do Ceará
}

Bezerra de Carvalho Ferreira, Glinton José; dos Santos, José Glauber Cavalcante; Carvalho de Vasconcelos, Alessandra

Avaliação do Controle Interno e Tamanho dos Órgãos sob Jurisdição do Tribunal de Contas do Ceará Administração Pública e Gestão Social, vol. 13, núm. 2, 2021

Universidade Federal de Viçosa, Brasil

Disponible en: http://www.redalyc.org/articulo.oa?id=351566014003

\section{(c) (1) 90}

Esta obra está bajo una Licencia Creative Commons Atribución-NoComercial-SinDerivar 3.0 Internacional. 


\title{
Avaliação do Controle Interno e Tamanho dos Órgãos sob Jurisdição do Tribunal de Contas do Ceará
}

\author{
Internal Control Assessment and Size of Agencies under the Jurisdiction of the Court of Auditors of Ceará \\ Evaluación del Control Interno y Tamaño de Los Órganos en Jurisdicción del Tribunal de Cuentas de Ceará \\ Glinton José Bezerra de Carvalho Ferreira \\ Redalyc: http://www.redalyc.org/articulo.oa? \\ Universidade Federal do Ceará, Brasil \\ id $=351566014003$
}

glinton@tce.ce.gov.br

José Glauber Cavalcante dos Santos

Universidade Federal do Ceará, Brasil

jglauber_cont@hotmail.com

Alessandra Carvalho de Vasconcelos

Universidade Federal do Ceará - UFC, Brasil

alevasconcelos.ufc@gmail.com

Recepción: 20 Enero 2020

Aprobación: 29 Enero 2020

Publicación: 01 Abril 2021

\section{Resumo:}

Objetivo da pesquisa: Analisar a relação entre a avaliação do controle interno e o tamanho dos órgãos jurisdicionados do Tribunal de Contas do Ceará (TCE/CE).

Enquadramento teórico: O estudo aborda a qualidade do controle interno em órgãos públicos assumindo a abordagem emanada pela teoria contingencial e explicando que influência exercem certas variáveis contingenciais (internas e externas) nessa estrutura organizacional.

Metodologia: O estudo analisa 57 entidades e utiliza dados de 2016. A avaliação do controle interno foi feita por meio dos formulários que constam na Instrução Normativa TCE/CE n. 03/2015, baseada no framework do COSO. A variável contingencial tamanho foi mensurada através do orçamento, despesa empenhada e do número de funcionários. O estudo é descritivo, documental e quantitativo: análise fatorial, testes de correlação e regressão quantílica.

Resultados: Foram identificados seis componentes do controle interno, sendo mais relevante a atuação e a responsabilidade da alta gestão. Os componentes mostram correlação significativa com o orçamento, a despesa empenhada e o número de funcionários. Existe influência negativa do tamanho dos órgãos na avaliação do controle interno.

Originalidade: Os resultados contribuem com a discussão acerca da atuação da gestão na definição de estratégias que amorteçam efeitos de contingências na estrutura das organizações públicas. A identificação de fatores contingenciais relevantes abre espaço para reflexões sobre a busca por adequação e eficiência no campo de estudos organizacionais das entidades públicas. O método da pesquisa também se apresenta como diferencial.

Contribuições teóricas e práticas: No aspecto gerencial, o funcionamento da administração pública é destaque, pois os resultados apontam caminhos para definir modelos de gestão mais realistas frente ao ambiente econômico, social, político e tecnológico. Na prática, sinaliza-se aos gestores a priorização dos controles internos focados em instrumentos de monitoramento, prevenção e correção de falhas. Resultados mais efetivos podem ser alcançados rapidamente, gerando economicidade de recursos.

PalaVRas-CHAVE: Teoria da contingência, Controle interno, Tamanho organizacional, Gestão pública, Tribunal de contas.

\section{Abstract:}

Objective: The objective of this paper is to evaluate the association between internal control assessment and the size of state government agencies under the jurisdiction of the Court of Auditors of Ceará (TCE/CE).

Theoretical framework: The internal control assessment of state government agencies was studied under the light of contingency theory, which allows to determine the influence of internal and external contingency variables on organizational structure.

Methods: The sample of this descriptive and quantitative desk study included 57 agencies from which information on internal control for the year 2016 was collected by TCE/CE using the COSO self-reporting framework, pursuant to normative directive n. 03/2015. The contingency variable 'agency size' combined budget, committed expenditure and number of staff. The data were submitted to factor analysis, correlation testing and quantile regression. 
Results: Six internal control components were identified, the most relevant being senior managerial performance and accountability. The components were significantly associated with budget, committed expenditure and number of staff. Agency size was negatively associated with internal control assessment.

Originality: Our results contribute to the discussion on the role of managerial performance in the formulation of strategies to mitigate contingency effects in the structure of government agencies. The identification of relevant contingency factors can subsidize further analyses of the quest for adequacy and efficiency in the field of organizational studies of public entities. The study is also unique regarding the methodology employed.

Theoretical and practical contributions: In managerial terms, our study focuses on the functioning of public administration, suggesting ways of defining more realistic management models to tackle current economic, social, political and technological challenges. In practical terms, managers are advised to prioritize internal controls focused on instruments of monitoring, prevention and correction of flaws. By doing so, greater effectiveness can be achieved in the short term, rationalizing resources.

KEYWORDS: Contingency theory, Internal control, Agency size, Public administration, Court of auditors.

\section{RESUMEN:}

Objetivo de la investigación: Analizar la relación entre la evaluación del control interno y el tamaño de los órganos jurisdiccionales del Tribunal de Cuentas de Ceará (TCE/CE).

Marco teórico: El estudio aborda la calidad del control interno en los organismos públicos adoptando el enfoque que emana de la teoría de la contingencia y explicando qué influencia tienen determinadas variables contingentes (internas y externas) en esta estructura organizativa.

Metodología: Este trabajo analiza 57 entidades y utiliza datos de 2016. La evaluación del control interno fue hecha a través de las formas constantes de la Instrucción Normativa TCE/CE n. 03/2015, con base en el referencial del COSO. La variable de contingencia tamaño de los órganos se midió a través del presupuesto y el gasto comprometido y el número de empleados. El estudio es descriptivo, documental y cuantitativo: análisis factorial, pruebas de correlación y regresión cuantil.

Resultados: Se identificaron seis componentes del control interno, siendo el rol y la responsabilidad de la alta gerencia el más relevante. Los componentes muestran una correlación significativa con el presupuesto, los gastos comprometidos y el número de empleados. Hay una influencia negativa del tamaño de los órganos en la evaluación del control interno.

Originalidad: Los resultados contribuyen a la discusión sobre el rol de la gestión en la definición de estrategias que mitiguen los efectos de las contingencias en la estructura de las organizaciones públicas. La identificación de factores contingentes relevantes abre un espacio para reflexiones sobre la búsqueda de adecuación y eficiencia en el campo de los estudios organizacionales de las entidades públicas. El método de investigación también se presenta como diferencial.

Contribuciones teóricas y prácticas: En el aspecto gerencial, se destaca el funcionamiento de la administración pública, ya que los resultados señalan formas de definir modelos de gestión más realistas frente al entorno económico, social, político y tecnológico. En la práctica, se aconseja a los gerentes que prioricen los controles internos enfocados en los instrumentos de monitoreo, prevención y corrección de fallas. Se pueden lograr resultados más efectivos rápidamente, generando ahorros de recursos.

Palabras ClaVe: Teoría de contingencia, Control interno, Tamaño organizacional, Gestión pública, Tribunal de cuentas.

\section{INTRODUÇÃO}

O controle interno é definido como conjunto de mecanismos estabelecidos com o objetivo de proteger ativos e recursos, impedir fraudes e detectar erros, gerando maior eficiência, precisão e confiabilidade nas ações da gestão (Beuren \& Zonatto, 2014; Choi, Lee, \& Sonu, 2013). Na administração pública, o controle interno tem função indispensável na legalidade dos atos dos gestores públicos, acumulando papel de supervisão (Barbieri \& Hortale, 2002; Beuren \& Zonatto, 2014).

Neste contexto, Diniz, Ribeiro, Libonati e Fragoso (2004) comentam que os Tribunais de Contas, entre as suas diversas prerrogativas de fiscalização, buscam verificar se o sistema de controle interno dos órgãos jurisdicionados está funcionando adequadamente e o grau de confiabilidade com que os relatórios contábeis produzidos podem fomentar a emissão de parecer sobre as prestaçóes de contas anuais.

Dessa forma, a existência de um adequado sistema de controle interno é imprescindível para a administração pública e para o sistema de controle externo, possibilitando exame prévio da situação do órgão jurisdicionado (Diniz, Ribeiro, Libonati, \& Fragoso, 2004). Diante disso, pode-se depreender que a compreensão dos sistemas de controle interno na gestão pública demanda maior investigação. A identificação 
de fatores que explicam os mecanismos de controle favorece a construção de um ambiente de monitoramento e gestão de risco mais eficaz (Beuren \& Zonatto, 2014).

Ademais, conforme elucidam Speeden e Perez (2020), a falta ou o fraco desempenho de instâncias de controle interno e externo impacta no planejamento e nas finanças públicas. Este estudo aborda a avaliação das estruturas de controle interno e sua relação com o tamanho dos órgãos, sendo este último fator contingencial segundo a teoria da contingência (Waterhouse \& Tiessen, 1978; Donaldson, 1999). Apesar de o controle interno desempenhar importante papel na gestão pública, há muito a se explorar em termos de contribuições científicas nesse campo de estudo (Beuren \& Zonatto, 2014).

Os sistemas de controle interno são vitais nas organizações, essencialmente na administração pública. Organizações públicas têm relevante papel social na oferta de serviços à comunidade e os recursos de que dispõem para cumprimento de sua finalidade demandam monitoramento (Barbieri \& Hortale, 2002; Beuren \& Zonatto, 2014; Choi et al., 2013).

Investigações nesse escopo são mais contributivas diante do recente cenário de crise política e de casos de fraude e (ou) corrupção na administração pública brasileira. Assim, informações sobre a avaliação do controle interno podem minimizar a sensação de impunidade, ineficiência e frágil controle e fiscalização de recursos públicos (Silva, Abreu, \& Couto, 2017).

Nesse sentido, importa aos Tribunais de Contas verificar se o sistema de controle interno dos órgãos jurisdicionados funciona adequadamente, gerando resultados satisfatórios por meio das políticas e normas instaladas, bem como se este auxilia o gestor público com informações que subsidiem a tomada de decisão.

A presente pesquisa amplifica a discussão acerca da interrelação entre as contingências ambientais, focando a contingência tamanho organizacional e a estrutura das organizaçóes do setor público. Se diversos fatores do ambiente externo e interno podem explicar estruturas e funções organizacionais, deve-se compreender como as variáveis contingenciais exercem essa interferência. Com isso, o controle e a gestão desses fatores ajudariam na eficiência da gestão dos recursos organizacionais, (Hickson, Hinings, Lee, Schneck, \& Pennings, 1971; Pugh, Hickson, Hinings, \& Turner, 1969).

Diante do exposto, tem-se como questão: como se caracteriza a relação entre a avaliação do controle interno e a variável contingencial tamanho dos órgãos jurisdicionados do Tribunal de Contas do Ceará (TCE/CE)?

O estudo objetiva analisar a relação entre a avaliação do controle interno e a variável contingencial relacionada ao tamanho dos órgãos jurisdicionados do Tribunal de Contas do Ceará (TCE/CE) e avaliar a estrutura de controle interno desses órgãos à luz da Instrução Normativa n. 03/2015.

A teoria da contingência sugere que não há estrutura organizacional única que seja efetiva para todas as organizações. Com isso, a otimização dessa estrutura varia de acordo com fatores contingenciais, destacandose o tamanho da organização (Waterhouse \& Tiessen, 1978; Donaldson, 1999). Relacionando-se a avaliação do controle interno e o tamanho dos órgãos jurisdicionados do TCE/CE, a pesquisa inova ao evidenciar como contingências impactam as estruturas das organizações públicas, ampliando a literatura sobre a atuação e o papel das Cortes de Contas no Brasil, ainda escassa segundo Loureiro, Teixeira e Moraes (2009).

Os resultados da pesquisa contribuem com a discussão acerca da atuação da gestão, na definição de estratégias que contrabalancem os efeitos das contingências na estrutura das organizações públicas. A identificação dos fatores contingenciais relevantes abre espaço para reflexões sobre a busca por adequação e eficiência no campo de estudos organizacionais das entidades públicas. Sobre isso, Silva e Mário (2015) incentivam estudos que compreendam os diversos aspectos relacionados ao controle nas organizações do setor público.

No aspecto gerencial, quanto ao funcionamento da administração pública, os resultados da pesquisa apontam caminhos para definir modelos de gestão mais realistas frente aos ambientes econômico, social, político e tecnológico (Santos, Bernardes, Rover, \& Mezzaroba, 2013), já que contemplariam melhor as dinâmicas de adequação baseando-se no desempenho. 
$\mathrm{Na}$ prática, esta pesquisa auxilia gestores na priorização de controles internos com foco nos instrumentos de monitoramento, prevenção e correção de falhas. Assim, resultados mais efetivos podem ser alcançados mais rápido, gerando economicidade de recursos.

Quanto à contribuição acadêmica na literatura sobre controle interno na gestão pública, de forma genérica, destaca-se a sustentação conceitual baseada na teoria da contingência. A pesquisa amplia estudos anteriores como Braga, Vasconcelos e De Luca (2013), que analisaram se os dispositivos de controle interno, segundo a Norma Brasileira de Contabilidade - NBC T 16.8, são contemplados nos relatórios anuais dos estados brasileiros; Cannavina e Parisi (2015), que identificaram, por meio da autoavaliação dos órgãos da Administração Pública Direta do Poder Executivo Federal, a percepção dos gestores sobre o quanto seus sistemas de controles internos têm sido utilizados para o alcance dos seus objetivos institucionais; e Souza e Silva (2016), que avaliaram a qualidade das práticas de controle interno evidenciadas no Relatório de Gestão, pelas Superintendências dos estados brasileiros, também com quadros de autoavaliação reportados ao Tribunal de Contas da União (TCU).

Ressalta-se o diferencial do trabalho no que tange à análise do relacionamento entre avaliação do controle interno e a variável tamanho organizacional em 57 entidades públicas à luz da teoria da contingência. Conforme Oliveira e Callado (2018), estudando organizações não governamentais (ONGs) brasileiras, a relação entre fatores contingenciais e as práticas referentes ao controle gerencial só foi investigada no Brasil em empresas com fins lucrativos, normalmente de grande porte. Portanto, existe uma lacuna de estudos que investiguem a relação dos fatores contingenciais nas estruturas de controle gerencial no setor público.

Ademais, Loureiro et al. (2009) explicam que a preocupação com o cidadão, com o maior controle por parte dos governantes e com o emprego de padrões mais democráticos de gestão pública foi integrada à cultura política brasileira. Assim, essa mudança teve reflexos na forma de trabalho das organizações públicas, que passaram a perseguir ferramentas gerenciais visando a aprimorar a gestão (Silva \& Mário, 2015). A presente pesquisa evidencia possíveis caminhos para a fiscalização da gestão pública por cidadãos e demais órgãos de fiscalização e (ou) controle.

A pesquisa estrutura-se em quatro seções, além da introdução: realiza-se a argumentação teórica relacionada à problemática e construção das hipóteses, em seguida, são descritos os procedimentos metodológicos em seguida. A quarta seção aponta resultados e discussão. Por fim, conclusões da pesquisa e sugestões a estudos futuros.

\section{DISCUSSÃO TEÓRICA E HIPÓTESE}

Compreender como são estruturados e avaliados os mecanismos de controle interno no âmbito das organizações, em especial as públicas, responsáveis por gerenciar e aplicar recursos públicos, pode ser determinante para a eficiência da gestão. Nesse sentido, torna-se pertinente investigar como o contexto no qual a entidade se insere reflete e influencia suas estruturas, de modo a auxiliar gestores a alcançar melhores resultados e responder à dinâmica da mudança.

Pugh et al. (1969) explicam que a estrutura das organizações está intimamente ligada ao contexto no qual operam. Como consequência, muitas das variações na estrutura organizacional acontecem devido à existência de fatores contextuais. Maletič, Maletič e Gomišček (2018) evidenciam o papel que as contingências ou fatores contingenciais têm no estabelecimento de práticas organizacionais, inclusive no desempenho da organização.

Os fatores contextuais são denominados contingências, aspectos do ambiente interno e externo que afetariam a estrutura da organização. Dentre as contingências, tamanho, tecnologia, função e a relação com outras organizações são sugeridos como antecedentes da mudança e do funcionamento organizacional (Pugh et al., 1969). Por sua vez, Oliveira e Callado (2018, p. 97) afirmam que "o fator contingencial tamanho ou 
porte inicia as discussões sobre os fatores contingenciais internos que podem afetar as práticas gerenciais de controle", sendo foco desta investigação.

Dallabona, Nardelli e Fernandes (2019) desenvolvem raciocínio semelhante afirmando que a teoria contingencial propõe que as estruturas organizacionais e os processos eficientes dependem do contexto da organização (Waterhouse \& Tiessen, 1978), sugerindo que variáveis contingenciais, como o tamanho, influenciam, entre os aspectos organizacionais, a utilização dos sistemas de controle gerencial.

Para Donaldson (1999), no campo dos estudos organizacionais, a abordagem contingencial fornece paradigma lógico que permite analisar a estrutura das organizações. As relações entre os membros de uma organização constituem sua estrutura. No escopo dessas relações estão os relacionamentos de autoridade e subordinação, os comportamentos requeridos pelos regulamentos da organização, inclusive os padrões na tomada de decisão, onde está o controle interno.

Pesquisas (Clinton, Pinello, \& Skaife, 2014; Kim \& Park, 2009; López \& Peters, 2010) mostram que a regulação possui contribuição relevante na institucionalização de práticas de controle interno. Além disso, nas organizações com maior publicidade das práticas de controle interno existe maior credibilidade. Assim, estudos como este ajudam os gestores que desejam melhorar a qualidade do controle interno, além de reguladores que buscam compreender esse processo de melhoria e os instrumentos de monitoramento (Liu, Lin, \& Shu, 2017).

Estudos internacionais (Woods, 2009; Shu, Chen, Lin, \& Chen, 2018; Chang, Chen, Cheng, \& Chi, 2019) indicam que existem fatores contingenciais internos e externos que podem influenciar os mecanismos de controle interno das organizações. Essas pesquisas consideram a teoria contingencial como uma abordagem sólida no que diz respeito à avaliação das estruturas de controle interno. Características como o capital humano, representado pela auditoria interna, o tamanho organizacional, além de regulamentações e a integridade corporativa tem influência sobre a qualidade dos controles internos (Woods, 2009; Shu et al., 2018; Chang et al., 2019).

A literatura documenta que as estruturas de controle interno podem ser influenciadas pelo tamanho das entidades, característica representada pelos recursos econômico-financeiros e humanos disponíveis (Barbieri \& Hortale, 2002; Choi et al., 2013; Henrique, Chagas, Guimarães, \& Leal, 2016; Liu et al., 2017; Monteiro, 2015; Queiroz, Nobre, Silva, \& Araújo, 2013; Silva, Celestino, Melo, \& Mól, 2017; Woods, 2009).

Barbieri e Hortale (2002) explicam que o controle tem a função de supervisionar as organizações e suas práticas, com a prerrogativa da busca por falhas e erros para que sejam realizadas correções. De acordo com Valentim, Silva e Passos (2016), a função do controle interno está relacionada com o objetivo de mitigação de riscos. No âmbito da administração pública, os riscos estão associados à utilização indevida de recursos públicos, desviando-se a finalidade do bem-estar social.

Nesse sentido, há uma relação próxima entre os mecanismos de controle e a avaliação de risco nas organizações. Essa avaliação é parte do processo de gerenciamento do risco, que tem por finalidade tomar decisões que reduzam as chances e os efeitos de eventos danosos para o patrimônio de uma entidade. Assim, a eficiência das organizações depende da adequação dos mecanismos de controle e do gerenciamento do risco, que consiste na identificação e avaliação dos riscos (Dervishi \& Kadriu, 2014; Solomon, Solomon, Norton, \& Joseph, 2000).

Ainda segundo Valentim et al. (2016), o nível de desempenho e o custo para a implantação e manutenção devem ser observados como ferramentas importantes ao processo de mensuração e controle das variáveis de risco na organização. Assim, os resultados esperados e os recursos são aspectos pertinentes para a eficiência do controle interno.

Segundo Dervishi e Kadriu (2014), sob elevado risco e incerteza os gestores tendem a se afastar do cumprimento de normas e regulamentos. Isso tende a ser prejudicial na administração pública, que gerencia recursos da sociedade e tem como um princípio básico a legalidade. Os mecanismos de controle tornam-se ainda mais relevantes nesse contexto, uma vez que a gestão dos riscos, possibilitada pelo controle interno, 
leva à maximização da eficiência, beneficiando a sociedade como parte interessada e reduzindo problemas financeiros (Solomon et al., 2000).

Capovilla e Gonçalves (2018) e Silva et al. (2017) evidenciam que o tamanho das organizações influencia seus mecanismos de controle e monitoramento. Conforme Capovilla e Gonçalves (2018), níveis iniciais de maturidade organizacional estão associados à fragilidade do ambiente de controle, especialmente no aspecto disseminação de padrões éticos. Silva et al. (2017) apontam relação positiva entre tamanho e o conjunto de instrumentos aplicados com intuito de alinhar interesses de gestores e stakeholders. $\mathrm{Na}$ avaliação do índice de eficiência de hospitais de alta complexidade do estado de São Paulo geridos por organizaçóes sociais, Tonelotto, Crozatti, Moraes e Righetto (2019) constataram que hospitais de maior porte e repasse recebido do estado e com maior quantidade de atendimentos são mais eficientes na gestão dos recursos. Segundo os autores, o efeito positivo no alcance dos indicadores de eficiência pode ser reflexo do estabelecimento de metas que devem ser atingidas pelas entidades e de seus mecanismos de controle interno.

Hickson et al. (1971) explicam a importância do gerenciamento estratégico das variáveis contingenciais, considerando-se a interferência dos fatores contextuais na estrutura das organizaçóes. Segundo os autores, são essas diferenças individuais que implicam em diferenças de desempenho organizacional (eficiência dos recursos e contingências relacionadas ao capital humano). Além disso, deve ser dada atenção ao contexto no qual ocorrem as diversas decisões e isso significa analisar a estrutura das organizações (Dervishi \& Kadriu, 2014). Essa estrutura compreende o tamanho, recursos, capital humano e mecanismos de controle diversos.

Sob enfoque da teoria contingencial, a estrutura das organizações se molda à complexidade das atividades e do ambiente organizacional interno e externo. Por essa razão, não há uma estrutura única que funcione para todas as organizações. A otimização das estruturas - como o controle interno - depende das contingências, como tamanho organizacional. As contingências refletem a influência que o ambiente exerce na organização (Donaldson, 1999).

O tamanho organizacional é uma variável contingencial que afeta a estrutura da organização no todo, inclusive a estrutura de controle (Taffarel, Almeida, Pereira, \& Clemente, 2018). Considera-se que quanto maior a estrutura financeira e (ou) orçamentária e de capital humano qualificado, maior a efetividade e eficiência do controle interno (Bryan, 2017; Chalmers, Hay, \& Khlif, 2018; Doyle, Ge, \& McVay, 2007; Liu et al., 2017; Lu \& Cao, 2018; Peterson, 2018).

Ji, Lu e Qu (2015) argumentam que a fragilidade dos controles internos depende de características como tamanho e complexidade. Liu et al. (2017) explicam que o capital humano pode ser ainda mais determinante que os recursos financeiros para a eficiência e eficácia organizacional. Por seu turno, Linsley e Shrives (2006) identificaram relação positiva entre o tamanho da organização e o volume de informações sobre risco. Postula-se que quanto maior a contingência tamanho, mais complexas as estruturas, que demandam melhores mecanismos de controle interno, corroborando Ji et al. (2015) e Liu et al. (2017).

Valentim et al. (2016) concluem que há diversos fatores a serem considerados para implementar ou fortalecer o processo de gestão e controle interno nas organizações. Segundo os autores, deve-se dar destaque, como barreiras detectadas para melhoria do controle interno, a cultura de gestão de risco, o quantitativo humano e a qualificação do capital humano.

Por este ângulo, Choi et al. (2013) indicaram que maiores investimentos em recursos humanos que integram o controle interno estão associados ao tamanho das organizações. Além do total de recursos aplicados na forma de ativos, o número de funcionários é sugerido como variável de tamanho. Liu et al. (2017) estabelecem que o capital humano exclusivo dos órgãos de controle interno desempenha papel importante na implementação dos mecanismos de controle, sendo os recursos humanos relevantes para a qualidade e desempenho do controle interno.

Embora os recursos econômico-financeiros e humanos disponíveis possam se impor como variáveis contingenciais da estrutura organizacional, essa relação possui diferenças. Doyle et al. (2007) analisaram determinantes da fragilidade do controle interno por meio do disclosure e constataram que as entidades 
com problemas mais sérios eram as menores, mais jovens e com dificuldade financeira. Porém, os recursos econômico-financeiros e a estrutura orçamentária são administrados pela gestão. Nesse sentido, Lu e Cao (2018) inferiram que o capital humano é importante variável no que se refere ao desempenho dos controles internos.

Do exposto, e replicando as duas proxies utilizadas na pesquisa internacional de Thomson (2010), em ONGs - faturamento (perspectiva econômico-financeira) e número de funcionários -, conjectura-se observar relação direta entre tamanho organizacional e práticas de controles gerenciais em organizações públicas.

Assim sendo, a presente pesquisa é diferenciada daquelas já realizadas pois explora o relacionamento da variável contingencial tamanho com a avaliação da estrutura de controle interno de órgãos e (ou) entidades públicas. Destarte, à luz da teoria da contingência, o estudo produz evidências da percepção de gestores públicos no que diz respeito ao tamanho organizacional considerando-se recursos econômico-financeiros e humanos disponíveis. Com isso, tem-se a seguinte hipótese do estudo:

H.: A variável contingencial tamanho organizacional afeta positivamente o controle interno dos órgãos e (ou) entidades públicas investigados.

$\mathrm{H}_{1 \mathrm{a}}$ : A variável contingencial tamanho, sendo mensurada pelos recursos econômico-financeiros, afeta positivamente o controle interno dos órgãos e (ou) entidades públicas.

$\mathrm{H}_{1 \mathrm{~b}}$ : A variável contingencial tamanho, sendo mensurada pelos recursos humanos, afetam positivamente o controle interno dos órgãos e (ou) entidades públicas.

\section{ASPECTOS METODOLÓGICOS}

A presente pesquisa tem natureza descritiva, uma vez que se põe a investigar fenômeno específico em um grupo particular de indivíduos. $\mathrm{O}$ estudo é classificado como documental, com uso de dados secundários (Collis \& Hussey, 2005), que segue abordagem quantitativa quanto ao tratamento dos dados (Roesch, 2006).

O estudo analisa dados dos órgãos jurisdicionados do Tribunal de Contas do Estado do Ceará (TCE/ CE) que enviaram prestação de contas referentes ao exercício de 2016. A prestação de contas desses órgãos possui formulário de avaliação da estrutura de controle interno padrão, conforme Quadro 1. Em acordo com a Instrução Normativa TCE/CE n. 03/2015, cada órgão realiza autoavaliação dos seus controles internos considerando 24 aspectos agrupados em 5 componentes. Essa estrutura de avaliação está alinhada ao framework estabelecido pelo Committee of Sponsoring Organizations of the Treadway Commission (COSO) (Pederneiras, Lopes, Ferreira, Santos, \& Ribeiro, 2018). A avaliação é feita no exercício seguinte a que se refere a prestação de contas e leva algum tempo para serem validadas as respostas dos órgãos. 
Quadro 1: Formulário de avaliação da estrutura de controle interno

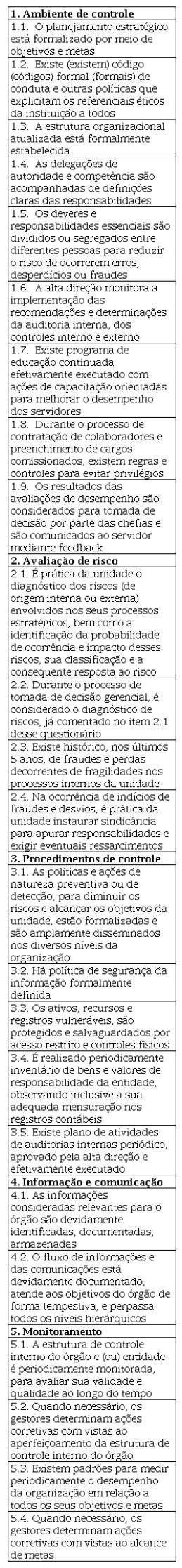


Fonte: Adaptado a partir da Instrução Normativa TCE/CE nº 03/2015.

$\mathrm{Na}$ autoavaliação, os órgãos atribuem notas de 1 a 4 (correspondendo a nunca, raramente, com frequência e sempre, respectivamente) considerando as afirmativas. A norma estabelece que as respostas aos aspectos determinam o nível de controle interno como satisfatório $(3 \leq$ Nota $\leq 4)$, razoável $(2 \leq$ Nota $<3)$ e insatisfatório $(1 \leq$ Nota $<2)$. Essa avaliação é um componente do risco dos órgãos levado em conta para realização de futuras auditorias. Nesse ponto, cabe mencionar que os estudos de Cannavina e Parisi (2015) e Souza e Silva (2016) também foram desenvolvidos a partir dos quadros de autoavaliação dos sistemas de controles internos. O primeiro avalia a percepção dos gestores dos órgãos da Administração Pública Direta do Poder Executivo Federal; o segundo com gestores das Unidades da Federação, ambos reportados ao Tribunal de Contas da União (TCU). Nota-se que a literatura ampara essa métrica de avaliação.

O envio do formulário de avaliação (Quadro 1) é obrigatório e a validação das repostas e a avaliação dos resultados passa por apreciação do corpo técnico do TCE/CE. A Instrução Normativa n. 03/2015 exige evidências documentadas que suportam as respostas (por exemplo, atas de reuniões, indicadores de desempenho ou mapeamento de processos). Havendo dúvidas acerca das respostas emitidas, das evidências confirmatórias apresentadas, ou, ainda, em caso de ausência de respostas, os devidos esclarecimentos são solicitados ao gestor responsável pelo órgão. Nessas ocasiões, a validação final das respostas somente será concluída na fase de reexame da matéria.

Ainda segundo a Instrução Normativa n. 03/2015, a norma tem harmonia com as disposições da International Organization of Supreme Audit Instructions (INTOSAI). O formulário é um instrumento de avaliação de eficiência dos órgãos sob jurisdição do TCE/CE que compreende o desenho e a estrutura do sistema de controle interno dos órgãos. Mesmo de natureza declaratória, a autoavaliação é sujeita à comprovação pelo TCE/CE. O gestor máximo de cada órgão é responsável pelas informações, que são acompanhadas de evidências comprobatórias. Conforme estabelece o parágrafo $2^{\circ}$, do art. $2^{\circ}$, da Instrução Normativa n. 03/2015, o gestor deve atestar a veracidade do conteúdo constante no formulário de avaliação.

Foram recebidas pelo TCE/CE 106 prestações de contas de vários órgãos jurisdicionados alusivas a 2016. O período se dá em razão da época de realização da pesquisa e da acessibilidade aos dados. Foram excluídos: (i) órgãos que possuíam a natureza jurídica de fundos (unidades orçamentárias sem controles próprios, vinculadas a outros órgãos da administração direta) - 33 órgãos e (ii) órgãos que não enviaram o formulário de autoavaliação do controle interno respondido junto às suas contas de gestão. Assim, compuseram a amostragem final 57 órgãos (identificados de 1 a 57). Os dados foram extraídos individualmente de 57 formulários que acompanham a prestação de contas dos órgãos sob jurisdição do TCE/CE, ou seja, órgãos estaduais e municipais que têm fiscalizadas e acompanhadas suas diversas ações pelo referido tribunal, agente de controle externo.

A amostragem é não-probabilística, compreendendo $54 \%$ da população inicial. Partindo-se dos órgãos que enviaram os formulários de autoavaliação, 90 entidades, esse percentual é 63\%. Portanto, as inferências apresentadas são pertinentes, porque abrangem parcela significativa das observações. São 53 órgãos do poder executivo, 2 órgãos do poder legislativo, 1 do poder judiciário e 1 vinculado ao Ministério Público. Desses, 32 são da administração direta e os demais são da administração indireta.

Fávero e Belfiore (2017) explicam que a amostragem não-probabilística é válida em função de características das unidades de análise e dificuldades na acessibilidade aos dados. Contudo, apontam os autores, as inferências devem ser prudentes no que se refere à generalização dos resultados. Em uma análise comparativa entre estados, outras conclusões podem ser evidenciadas em estudos posteriores.

Outros estudos quantitativos recorreram a uma estratégia semelhante àquela utilizada nesta pesquisa, citando-se Mazulo et al. (2013) no Exército Brasileiro, Queiroz et al. (2013) nos municípios do Rio Grande do Norte, e Taffarel et al. (2008) no segmento madeireiro do Paraná. Sob diferente perspectiva, adotando a estratégia do estudo de caso, Alves e Moraes (2016), Capovilla e Gonçalves (2018), Henrique, Chagas, 
Guimarães e Leal (2016), Lino, Carvalho, Aquino e Azevedo (2019) e Piccoli, Balestrim e Rover (2015) desenvolveram casos sobre controle interno no setor público.

De posse dos formulários de cada órgão, aplicou-se a técnica estatística de análise fatorial por componentes principais. A análise fatorial foi empregada buscando-se dois objetivos: (1) identificar dimensões latentes de agrupamento dos 24 aspectos do formulário de avaliação da estrutura de controle interno; e (2) estabelecer um ranking com a avaliação do controle interno dos órgãos, baseado nas dimensões latentes identificadas. O score de avaliação dos órgãos obtido na análise fatorial para o ranking é a primeira variável de interesse, denominada

O ranking ordena os órgãos investigados segundo a avaliação da estrutura de controle interno. Ele é obtido pela soma dos valores de cada fator ponderada pela sua variância. Assim, podem ser identificados os órgãos jurisdicionados do TCE/CE com melhor avaliação do controle interno por meio dos fatores mais promissores para avaliação da estrutura de controle interno (autovalor $>1,000$ ).

Para Fávero e Belfiore (2017), esse é um critério bastante aceito e empregado para a formação de rankings. Neste caso, o objetivo é estabelecer a nota de avaliação do controle interno, dispondo os órgãos com maiores e menores notas. Esse critério assume a avaliação da estrutura do controle interno em todas as variáveis originais a partir da Instrução Normativa n. 03/2015. Se apenas um fator fosse considerado, equivocadamente não se levaria em conta o resultado obtido em determinada variável "que eventualmente compartilhe um considerável percentual de variância com o segundo fator” (Fávero \& Belfiore, 2017, p. 406).

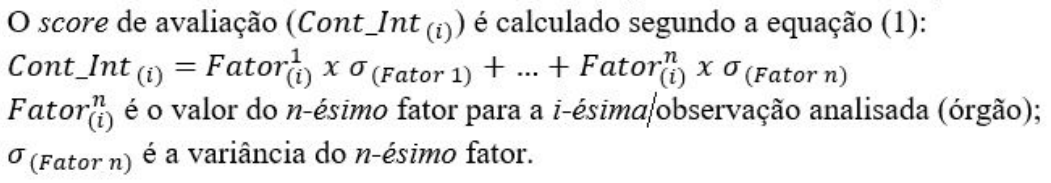

A segunda variável de interesse é o tamanho dos órgãos. A literatura sugere que a variável tamanho organizacional pode estar associada à complexidade das estruturas de governança e sistemas de controle gerencial (Dallabona et al., 2019; Oliveira \& Callado, 2018; Thomson, 2010) de entidades públicas e privadas. O tamanho é comumente representado pelos recursos econômico-financeiros e humanos à disposição das entidades (Barbieri \& Hortale, 2002; Choi et al., 2013; Henrique et al., 2016; Liu et al., 2017; Monteiro, 2015; Queiroz et al., 2013; Silva, Celestino et al., 2017; Thomson, 2010).

Sendo assim, essa medida foi analisada sob duas perspectivas: (1) econômico-financeira (orçamento previsto e despesa empenhada) e (2) recursos humanos (total de servidores e servidores ativos por órgão). As proxies são $O r c ̧ a_{(i)}$ (logaritmo natural do montante orçado), Desp_Emp(i) (logaritmo natural do montante empenhado), $R h_{(i)}$ (logaritmo natural do total de funcionários ativos e não ativos de cada órgão: servidores ativos, aposentados, pensionistas, estagiários e bolsistas) e Serv_Ativo (i) (logaritmo natural do número de servidores ativos).

Sobre as proxies referentes a recursos humanos, destaca-se que o quantitativo relacionado aos recursos humanos de cada órgão é relatado pelo total, composto pelos servidores ativos, aposentados, pensionistas, estagiários e bolsistas. Essa disposição ocorre porque os órgãos possuem dotação orçamentária específica para remuneração dos funcionários e ex-funcionários. Logo, considerou-se apenas o valor total de funcionários de cada órgão $R h_{(i)}$ e de servidores ativos Serv_Ativo(i), essa última considera o capital humano permanente.

Foram realizados testes de correlação entre o score de avaliação do controle interno dos órgãos (Cont_Int (i) ), obtido na análise fatorial, e as proxies de tamanho dos órgãos. Foram feitas estimações por meio de regressão quantílica, que emprega os quantis e para estimar os coeficientes lineares. Nesse caso, Cont_Int (i) é variável dependente; Orça(i), Desp_Emp(i), $R h_{(i)}$, Serv_Ativo $(i)$ são independentes. Os dados foram analisados com auxílio dos softwares Statistical Package for the Social Sciences $\left(\mathrm{SPSS}^{\circ}\right)$ e STATA . 


\section{ANÁLISE DOS RESULTADOS}

\subsection{Análise descritiva}

A Tabela 1 contribui com a caracterização da amostra por meio das notas de avaliação da estrutura de controle interno dos órgãos jurisdicionados do TCE/CE. A análise considera as notas por unidade orçamentária e por aspecto do formulário de avaliação.

Tabela 1: Avaliação dos controles internos por órgão e por item do formulário

\begin{tabular}{|c|c|c|c|c|c|c|}
\hline $\begin{array}{l}\text { Unidade } \\
\text { orçamentária }\end{array}$ & Média & Mínimo & Máximo & Poder & Administração & Natureza \\
\hline \multicolumn{7}{|c|}{ Painel A: 3 maiores médias de avaliação } \\
\hline CGE & 3,8750 & 3 & 4 & Executivo & Direta & $\begin{array}{l}\text { Adm. } \\
\text { Direta }\end{array}$ \\
\hline $\mathrm{TCM}-\mathrm{CE}$ & 3,8333 & 2 & 4 & Legislativo & Direta & $\begin{array}{l}\text { Adm. } \\
\text { Direta }\end{array}$ \\
\hline FUNCEME & 3,7500 & 1 & 4 & Executivo & Indireta & Fundação \\
\hline \multicolumn{7}{|c|}{ Painel A: 3 menores médias de avaliação } \\
\hline $\begin{array}{l}\text { Secretaria de } \\
\text { Des. } \\
\text { Econômico }\end{array}$ & 1,3750 & 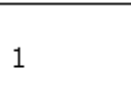 & 4 & Executivo & Direta & $\begin{array}{l}\text { Adm. } \\
\text { Direta }\end{array}$ \\
\hline $\begin{array}{l}\text { Junta } \\
\text { Comercial do } \\
\text { Estado do } \\
\text { Ceará }\end{array}$ & 1,1250 & 1 & 4 & Executivo & Indireta & Autarquia \\
\hline $\begin{array}{l}\text { Cia. de } \\
\text { Habitação do } \\
\text { Ceará }\end{array}$ & 1,0000 & 1 & 1 & Executivo & Indireta & $\begin{array}{l}\text { Empresa } \\
\text { Pública }\end{array}$ \\
\hline $\begin{array}{l}\text { Itens do } \\
\text { formulário } \\
\text { de avaliação }\end{array}$ & Média & Mínimo & Máximo & & & \\
\hline \multicolumn{7}{|c|}{ Painel A: 3 maiores médias de avaliação } \\
\hline Item 1.3 & 3,6667 & 1 & 4 & & & \\
\hline Item 1.4 & 3,6316 & 1 & 4 & & & \\
\hline Item 2.4 & 3,3509 & 1 & 4 & & & \\
\hline \multicolumn{7}{|c|}{ Painel B: 3 menores médias de avaliação } \\
\hline Item 5.1 & 1,8947 & 1 & 4 & & & \\
\hline Item 2.1 & 1,8772 & 1 & 4 & & & \\
\hline Item 2.2 & 1,8070 & 1 & 4 & & & \\
\hline
\end{tabular}

Fonte: Elaboração própria.

CGE (Controladoria e Ouvidoria Geral do Estado), TCM-CE (Tribunal de Contas dos Municípios), FUNCEME (Fundação Cearense de Meteorologia e Recursos Hídricos).

O primeiro estrato da Tabela 1 aponta dois órgãos de atividades contábeis de fiscalização e controle com melhor média de avaliação, a Controladoria e Ouvidoria Geral do Estado (CGE) e o Tribunal de Contas dos Municípios (TCM-CE). Destaca-se que, embora a Controladoria e a Ouvidoria possuam diferentes funções que se complementam, essa última não detém papel em atividades contábeis, coexistindo no mesmo órgão jurisdicionado da Controladoria. Tendo em conta os órgãos de melhor avaliação, o resultado parece condizente com a sua atuação. A Fundação Cearense de Meteorologia e Recursos Hídricos (FUNCEME) é um órgão estadual voltado à pesquisa e desenvolvimento em meteorologia que, apesar de não relacionado com as atividades de controle próprias da contabilidade, apresentou a terceira melhor média.

Dentre os 57 órgãos analisados através dos formulários, 19 (33\%) apresentaram nota média $\geq 3$, que para a Instrução Normativa TCE/CE n. 03/2015 indica avaliação satisfatória do controle interno. Órgãos com as menores médias $(1 \leq$ Nota $<2)$ foram $8(14 \%)$, enquanto aqueles com avaliação razoável $(2 \leq$ Nota 
< 3) somaram 30 (53\%). Esses resultados sugerem preocupação quanto à estrutura de controle interno, porque parcela representativa $(67 \%)$ dos órgãos jurisdicionados participantes do estudo dispõe de avaliação insatisfatória ou razoável.

Ressalta-se que a avaliação é individualizada por aspecto e isso sugere a heterogeneidade e o grau de maturidade das estruturas de controle interno nesses órgãos. A unidade orçamentária que detém a terceira maior média global teve, ao menos, um aspecto avaliado insatisfatório.

$\mathrm{Na}$ análise descritiva por item, destacaram-se positivamente os aspectos voltados à estrutura organizacional, delegação de autoridades e competências, além da apuração de fraudes e desvios (itens 1.3, 1.4 e 2.4). Os aspectos com avaliação insatisfatória refletem a eficiência da estrutura de controle interno e a avaliação de riscos. Conforme a literatura sobre avaliação de riscos (Dervishi \& Kadriu, 2014; Linsley \& Shrives, 2006; Salomon et al., 2000), o valor médio dos itens 5.1, 2.1 e 2.2 pode ser prejudicial para a gestão desses órgãos, porque são indicadores que refletirão negativamente na eficiência dos recursos. A fragilidade dos controles internos e da avaliação de riscos pode incentivar desvios de finalidade dos gestores e demais agentes, além de subdimensionar fatores de risco.

\subsection{Análise fatorial}

Para a análise fatorial, considerando os 24 aspectos da autoavaliação dos controles internos, foi verificada a adequação global da amostragem pela estatística Kaiser-Meyer-Olkin (KMO) e teste de esfericidade de Bartlett. A estatística KMO $(0,736)$ apontou que os aspectos analisados compartilhavam variância considerável para a formação de fatores. O teste de esfericidade de Bartlett $(0,000)$, significativo ao nível de $1 \%$, indicou que as correlações entre os aspectos são diferentes de zero, permitindo a extração de fatores.

A adequação individual dos 24 aspectos se deu por meio da análise da matriz anti-imagem e das comunalidades. A medida de adequação de amostragem para cada item oscilou entre 0,525 (item 2.3) e 0,904 (item 3.5). A comunalidade de cada item variou entre 0,464 (item 1.6) e 0,841 (item 2.1). A análise da adequação individual também foi considerada satisfatória para a extração de fatores.

Aplicando-se o método de extração por componentes principais, assumindo-se autovalores maiores que 1,000 e a rotação Varimax para os fatores, foi possível extrair 6 componentes, sendo 68,6\% a variância total explicada. A Tabela 2 sumariza os resultados da análise fatorial incorporando os aspectos da autoavaliação dos controles internos aos fatores indicados. 
Tabela 2: Componentes extraídos da análise fatorial

\begin{tabular}{|c|c|c|c|c|}
\hline Componentes & $\begin{array}{l}\text { Definição com } \\
\text { base na } \\
\text { descrição dos } \\
\text { itens }\end{array}$ & Autovalor & Variância & $\begin{array}{l}\text { Itens do formulário } \\
\text { de autoavaliação } \\
\text { (carga fatorial) }\end{array}$ \\
\hline 1 & $\begin{array}{l}\text { Atuação e } \\
\text { responsabilidades } \\
\text { da alta gestão }\end{array}$ & 8,209 & $34,21 \%$ & $\begin{array}{l}1.6(0,582) ; 1.8 \\
(0,557) ; 1.9(0,568) ; \\
3.4(0,585) ; 3.5 \\
(0,581) ; 4.2(0,697) ; \\
5.2(0,604) ; 5.3 \\
(0,710) \text { e } 5.4(0,726)\end{array}$ \\
\hline 2 & $\begin{array}{l}\text { Estrutura de } \\
\text { avaliação de } \\
\text { riscos }\end{array}$ & 2,287 & $9,529 \%$ & $\begin{array}{l}2.1(0,830) ; 2.2 \\
(0,807) ; 3.1(0,527) \mathrm{e} \\
5.1(0,678)\end{array}$ \\
\hline 3 & $\begin{array}{l}\text { Plano de longo } \\
\text { prazo, } \\
\text { informação e } \\
\text { qualificação }\end{array}$ & 1,914 & $7,976 \%$ & $\begin{array}{l}1.1(0,808) ; 1.7 \\
(0,519) ; 3.2(0,707) \\
3.3(0,839) \text { e } 4.1 \\
(0,601)\end{array}$ \\
\hline 4 & $\begin{array}{l}\text { Conduta e } \\
\text { delegaçấo de } \\
\text { competências }\end{array}$ & 1,687 & $7,031 \%$ & $\begin{array}{l}1.2(0,816) ; 1.4 \\
(0,571) \text { e } 1.5(0,705)\end{array}$ \\
\hline 5 & $\begin{array}{l}\text { Monitoramento e } \\
\text { combate a } \\
\text { fraudes }\end{array}$ & 1,213 & $5,053 \%$ & $\begin{array}{l}2.3(0,846) \text { e } 2.4 \\
(0,728)\end{array}$ \\
\hline 6 & $\begin{array}{l}\text { Estrutura } \\
\text { organizacional }\end{array}$ & 1,153 & $4,803 \%$ & $1.3(0,787)$ \\
\hline
\end{tabular}

Fonte: Elaboração própria.

Os resultados sugerem a identificação de 6 varáveis latentes da avaliação do controle interno. Esses fatores não coincidem com a estrutura do formulário baseado nos componentes do framework do COSO. Valentim et al. (2016) demonstram que muitas pesquisas apresentam como instrumento de controle e gestão o framework do COSO, mas outros aspectos devem ser considerados para aperfeiçoamento do modelo, especialmente quanto à gestão de riscos, por exemplo, a governança como mecanismo de alinhamento de interesses e redução da assimetria informacional (Valentin et al. 2016). A literatura também indica que muita atenção deve ser dada à geração de informações, proposição de objetivos e reação ao risco (Valentin et al., 2016; Silva et al., 2017).

Segundo Silva, Couto et al. (2017), o quantitativo de normas que aprimoram o controle interno nos órgãos públicos cresceu entre 2003 e 2016, após o anúncio da Operação Lava Jato. Foram criados mecanismos de controle e de revisão da estrutura de controle da administração pública (Lei da Anticorrupção, Lei de Acesso à Informação e a criação do Programa de Integridade na gestão pública). Esse aporte legal aperfeiçoa as práticas de controle interno com base na estrutura do COSO, principalmente em relação a "ambiente de controle" e "informação e comunicação" (Silva et al., 2017).

A análise fatorial permitiu identificar os fatores mais promissores para a avaliação do controle interno desse órgão. A Tabela 2 relata a denominação atribuída às variáveis latentes extraídas pela análise fatorial com base na descrição dos aspectos do formulário da Instrução Normativa TCE/CE n. 03/2015.

O componente mais expressivo na avaliação do controle interno tem variância explicada de $34 \%$ e incorpora 9 aspectos. Juntos os itens 1.6, 1.8, 1.9, 3.4, 3.5, 4.2, 5.2, 5.3 e 5.4 abordam a atuação direta ou indireta dos gestores na promoção e disseminação dos instrumentos de controle interno e suas responsabilidades e responsabilização por atos; o Fator 1 é denominado "atuação e responsabilidades da alta gestão”.

O segundo componente inclui os itens 2.1, 2.2, 3.1 e 5.1 e possui quase $10 \%$ de variância explicada, denominado "estrutura de avaliação de riscos". O formulário de avaliação possui o componente "avaliação de riscos", mas a análise fatorial não considerou os itens 2.3 e 2.4 na composição da variável latente. Em seus 
lugares os itens 3.1 e 5.1, que tratam da formalização das ações de combate e detecção de riscos, além da atualização e revisão do plano de ações. A avaliação dos riscos e seu gerenciamento são ressaltados por diversos estudos como importante elemento do controle interno (Dervishi \& Kadriu, 2014; Linsley \& Shrives, 2006; Salomon et al., 2000).

O terceiro componente intitula-se "plano de longo prazo, informação e qualificação". Ele comporta 5 aspectos (itens 1.1, 1.7, 3.2, 3.3 e 4.1), explicando quase $8 \%$ da avaliação do controle interno. Além do planejamento estratégico, esse fator incorpora a segurança e o sigilo das informações, juntamente com a prerrogativa de qualificação contínua dos servidores.

O quarto componente foi denominado "conduta e delegação de competências", com 7\% da variância explicada. Apenas três itens (1.2, 1.4 e 1.5) constituem esse fator que trata dos aspectos comportamentais dos membros que compõem o órgão: valores éticos, competências, delegação de poder e segregação de atribuições. Monteiro (2015) cita que entre as principais barreiras para avaliação e implementação de um sistema de controle interno estão a carência de pessoal em número e qualidade técnica adequadas, corroborando a evidência observada.

O quinto componente inclui os itens 2.3 e 2.4: "monitoramento e o combate a fraudes". A análise fatorial sugere separar ações que antecedem comportamentos de risco, voltadas ao planejamento inicial (Fator 2) e ações de acompanhamento e de repressão aos desvios de conduta (Fator 5). O último componente isolou o item 1.3 do formulário utilizado pelos órgãos na avaliação da estrutura do controle interno. Esse aspecto trata da atualização da estrutura organizacional, aparentemente pouco relacionado aos demais.

Após a extração dos componentes, elaborou-se um ranking apresentado na Tabela 3 por meio da avaliação da estrutura de controle interno baseado na equação (1).

Tabela 3. Scores fatoriais por unidade orçamentária

\begin{tabular}{|c|c|c|c|c|c|c|c|}
\hline \multirow{2}{*}{ Unidade } & \multicolumn{6}{|c|}{ Fatores e Scores } & \multirow[t]{2}{*}{ Total } \\
\hline & 1 & 2 & 3 & 4 & 5 & 6 & \\
\hline \multicolumn{8}{|c|}{ Painel A: maiores scores } \\
\hline CGE & 1,39 & 1,07 & 0,45 & 0,50 & 0,50 & $-0,11$ & 0,670 \\
\hline Cagece & 1,66 & $-0,50$ & 0,65 & 0,74 & 0,07 & $-0,96$ & 0,583 \\
\hline $\mathrm{TCM}-\mathrm{Ce}$ & 0,89 & 1,87 & 0,52 & $-0,22$ & 0,57 & 0,23 & 0,550 \\
\hline \multicolumn{8}{|c|}{ Painel B: menores scores } \\
\hline \multicolumn{8}{|c|}{$\begin{array}{l}\text { Secretaria } \\
\text { do Des. }\end{array}$} \\
\hline $\begin{array}{l}\text { Econômico } \\
\text { Cia. de }\end{array}$ & $-0,88$ & 0,09 & $-1,78$ & $-1,98$ & 0,41 & $-0,52$ & $-0,579$ \\
\hline $\begin{array}{l}\text { Habitação } \\
\text { do Ceará } \\
\text { Junta } \\
\text { Comercial } \\
\text { do Estado }\end{array}$ & $-0,60$ & $-0,01$ & $-1,54$ & $-1,69$ & $-1,33$ & $-2,46$ & $-0,635$ \\
\hline do Ceará & $-0,99$ & 0,37 & $-1,63$ & $-1,92$ & $-1,67$ & $-0,45$ & $-0,675$ \\
\hline
\end{tabular}

Fonte: Elaboração própria.

CGE (Controladoria e Ouvidoria Geral do Estado), Cagece (Companhia de

Água e Esgoto do Ceará), TCM-CE (Tribunal de Contas dos Municípios).

A Tabela 3 indica os três órgãos com melhor/menor avaliação, portanto, melhor estrutura do controle interno/estrutura mais frágil do controle interno. Diferentemente dos resultados da Tabela 1 , baseados apenas na média simples da avaliação, a Tabela 3 indica as inferências que são baseadas apenas nos seis fatores extraídos e os respectivos scores fatoriais.

Os scores fatoriais confirmam a boa avaliação da Controladoria e Ouvidoria Geral do Estado e do Tribunal de Contas dos Municípios. Na análise fatorial a FUNCEME perde espaço para a Companhia de Água e Esgoto do Ceará, que tem a segunda melhor avaliação da amostra. A FUNCEME cai para $16^{a}$ posição com 
a extração dos fatores. Os órgãos com menor avaliação com base nos scores fatoriais coincidem com aqueles identificados pela avaliação baseada nas notas médias. Esses resultados parecem reforçar a ideia de que as características desses órgãos acabam por refletir sobre o controle interno, algo preconizado pela abordagem contingencial (Woods, 2009; Shu et al., 2018; Chang et al., 2019).

Isso fica claro nas colocações de Capovilla e Gonçalves (2018) e Silva et al. (2017), segundo os quais aspectos como o tamanho e a própria maturidade da organização têm alguma influência nos mecanismos de controle interno, inclusive na sua fragilidade. Deve-se citar que órgãos como Defensoria Pública Geral do Estado e Escola de Gestão Púbica do Estado do Ceará aparecem entre as oito melhores avaliações.

\subsection{Análise de correlação e regressão quantílica}

A Tabela 4 apresenta os resultados dos testes de correlação e da estimação dos modelos quantílicos considerando a avaliação da estrutura de controle interno e o tamanho dos órgãos. Não há correlação estatisticamente significante entre o score de avaliação do controle interno dos órgãos e as proxies de tamanho dos órgãos. Contudo, há correlação estatisticamente significante analisando-se cada componente, fato que ratifica a importância da análise fatorial.

Tabela 4: Resultados dos testes de correlação e da regressão quantílica

Tabela 4: Resultados dos testes de correlação e da regressão quantílica

\begin{tabular}{|c|c|c|c|c|}
\hline \multicolumn{5}{|c|}{ Painel A: Correlação } \\
\hline & $0,118^{(a)}$ & $0,190^{(a)}$ & $0,155^{(a)}$ & $0,198^{(a)}$ \\
\hline Fator $1_{(i)}$ & $0,118^{(a)}$ & $0,175^{(a)}$ & $0,143^{(a)}$ & $0,167^{(a)}$ \\
\hline Fator ${ }^{2}$ (i) & $\begin{array}{l}-0,164 \\
\text { (b) }\end{array}$ & $\begin{array}{l}-0,138 \\
\text { (b) }\end{array}$ & $\begin{array}{l}-0,079 \\
\text { (b) }\end{array}$ & $\begin{array}{l}-0,030 \\
\text { (b) }\end{array}$ \\
\hline Fator $3_{(i)}$ & $\begin{array}{l}0,221^{(b)} \\
\times\end{array}$ & $\begin{array}{l}0,281^{(b)} \\
* *\end{array}$ & $0,117^{(b)}$ & $0,146^{(b)}$ \\
\hline Fator $4_{(i)}$ & $0,130^{\text {(b) }}$ & $0,160^{(b)}$ & $0,098^{(b)}$ & $0,125^{(b)}$ \\
\hline Fator 5 (i) & $0,014^{(b)}$ & $\begin{array}{l}-0,044 \\
\text { (b) }\end{array}$ & $\begin{array}{l}-0,067 \\
\text { (b) }\end{array}$ & $\begin{array}{l}-0,065 \\
\text { (b) }\end{array}$ \\
\hline Fator $6_{(i)}$ & $\begin{array}{l}-0,303 \\
\text { (b) } * *\end{array}$ & $\begin{array}{l}-0,222 \\
\text { (b) }\end{array}$ & $\begin{array}{l}-0,256 \\
\text { (b) } *\end{array}$ & $\begin{array}{l}-0,202 \\
\text { (b) }\end{array}$ \\
\hline Observações & 57 & $50^{(c)}$ & $56^{(\mathrm{c})}$ & $56^{(\mathrm{c})}$ \\
\hline \multicolumn{5}{|c|}{$\begin{array}{l}\text { Painel B: Regressão Quantílica } \\
\text { Variáveis } \\
\text { independentes (dependente) }\end{array}$} \\
\hline \multirow[t]{5}{*}{ Constante } & 0,046 & $0,117 * *$ & $0,080 *$ & $0,109^{* *}$ \\
\hline & $-0,802$ & - & - & - \\
\hline & - & $\begin{array}{l}-2,145 \\
* *\end{array}$ & - & - \\
\hline & $\overline{-}$ & - & $-0,443^{*}$ & - \\
\hline & - & - & - & $\begin{array}{l}-0,552 \\
* *\end{array}$ \\
\hline $\begin{array}{l}\text { Pseudo } R^{2} \\
\text { Observações }\end{array}$ & $\begin{array}{l}0,006 \\
57 \\
\end{array}$ & $\begin{array}{l}0,052 \\
50\end{array}$ & $\begin{array}{l}0,026 \\
56\end{array}$ & $\begin{array}{l}0,043 \\
56\end{array}$ \\
\hline
\end{tabular}

Fonte: Elaboração própria.

a) Correlação de Pearson (medidas paramétricas), (b) Correlação de Spearman (medidas não paramétricas),

(c) 7 órgãos não informaram o valor da despesa empenhada e apenas um órgão não disponibilizou o número de funcionários, ${ }^{*}$ correlação significante ao nível de $10 \%,{ }^{* *}$ correlação significante ao nível de $5 \%$. 
O Fator 3, "Plano de longo prazo, informação e qualificação", apresentou correlação positiva com Orça (i) (p-value < 0,10) e Desp_Emp (i) ( p-value < 0,05). Pode-se sugerir que maiores avaliações no Fator 3 poderão estar acompanhadas do incremento do orçamento e da despesa empenhada. São observadas correlações estatisticamente significantes e negativas com o Fator 6, "Estrutura organizacional", Orça (i) (p-value < 0,05) e $R h_{\text {(i) }}$ (p-value $\left.<0,10\right)$. Nesse caso, acréscimos no valor orçado ou no quantitativo de servidores podem ser acompanhados de uma menor avaliação da estrutura de controle interno dos órgãos. Os resultados dos testes de correlação têm alinhamento com as evidências obtidas por Chang et al. (2019), Woods (2009), Shu et al. (2018), principalmente quanto ao Fator 3. Segundo Woods (2009), a informação e a comunicação são importantes atributos na estrutura do sistema de controle interno, sendo isso válido para organizações públicas.

Os resultados das análises com regressões quantílicas apontam que as proxies de tamanho Desp_Emp(i), $R h$ (i) Serv_Ativo (i) afetam o score de avaliação do controle interno dos órgãos (Cont_Int (i)). Sugere-se que há efeito negativo do tamanho organizacional na avaliação no controle interno. Sinaliza-se com isso que órgãos menores têm melhor avaliação, principalmente quando a proxy é a despesa empenhada. Mesmo com menores gastos e menor quantitativo de funcionários, esses órgãos demonstram melhores mecanismos de controle interno. Essas conclusões corroboram aquelas obtidas nos estudos de Liu et al. (2017), mas divergem de Lu e Cao (2018) e Thomson (2010). No caso dos recursos humanos, pode-se ter como pressuposição a ideia de que um número muito elevado de participantes pode não ajudar o monitoramento.

\section{DISCUSSÃO DOS RESULTADOS}

As inferências alcançadas com a análise fatorial podem ser importantes para a revisão dos aspectos de autoavaliação dos controles internos dos órgãos do TCE/CE. A extração de 6 fatores sugere que podem existir outras dimensões de avaliação da estrutura de controle interno diferentes do COSO. Deve-se atentar para a atuação e responsabilidades da gestão como componente preponderante na análise fatorial. Isso corrobora as contribuições e os resultados de pesquisas que ressaltam o papel do componente humano na eficiência dos mecanismos de controle interno das organizações (Woods, 2009; Shu et al., 2018; Chang et al., 2019).

Silva et al. (2017) e Valentim et al. (2016) ajudam a compreender os resultados da pesquisa quando sugerem que o framework do COSO deveria ser aplicado levando-se em consideração as características das entidades e o contexto em que atuam. Pederneiras et al. (2018) explicam que o controle depende de variáveis econômicas, sociais e comportamentais. A qualidade na aplicação das práticas do controle interno, por sua vez, depende de aspectos como estrutura e funcionalidade, além da competência dos agentes.

Esse resultado reforça a importância de lideranças gerenciais nos órgãos públicos como referência comportamental de eficiência e boa administração dos recursos (Mazulo, Vieira, \& Silva, 2013). Capovilla e Gonçalves (2018) reforçam a ideia da contribuição das competências de liderança gerencial na implementação de estratégias com foco na melhoria do controle interno e disseminação de padrões éticos.

Os resultados da análise quantitativa levam à rejeição da hipótese geral $\mathrm{H} 1$ e das hipóteses específicas $\mathrm{H} 1 \mathrm{a}$ e $\mathrm{H} 1 \mathrm{~b}$. Segundo elas, a qual a variável contingencial tamanho organizacional, mensurada com base nos recursos econômico-financeiros e humanos, afetava positivamente o controle interno, o que não foi confirmado. Os resultados podem indicar que o tamanho desses órgãos não é compatível com a estrutura de controle interno, indicando que, na verdade, órgãos menos “inchados" poderão ser mais eficientes. Diferente do esperado, à luz da teoria contingencial, a relação negativa indicada na regressão quantílica pode sugerir que melhores estruturas de controle não estão relacionadas ao maior volume de recursos econômico-financeiros e humanos à disposição das entidades. Nesse caso, órgãos com despesa empenhada inferior e menor quantitativo de funcionários apresentaram melhor avaliação do controle interno. À vista disso, pode-se conjecturar que uma maior eficiência e eficácia dos controles internos pode estar relacionada à otimização dos recursos financeiros e humanos nessas unidades orçamentárias. Apesar de contrapor parte da literatura sobre o tema, os resultados 
são coerentes com a lógica de aprimoramento da estrutura organizacional, ideia da teoria contingencial (Dallabona et al., 2019; Donaldson, 1999; Hickson et al., 1971; Oliveira \& Callado, 2018; Pugh et al., 1969; Waterhouse \& Tiessen, 1978).

A relação esperada se manifesta em componentes particulares da estrutura de controle interno dos órgãos, como mostrou a correlação. Assim, é possível explorar os aspectos de modo individual ou em pequenos grupos visando o aprimoramento da eficiência dos mecanismos de controle na sua totalidade. Essa evidência é útil para a atuação de gestores públicos das unidades orçamentárias analisadas. Como ficou constatado, existem fatores específicos que têm relação com a avaliação dada aos controles internos, caso daqueles referentes à plano de longo prazo, informação e qualificação (relação positiva) e estrutura (relação negativa).

Nesses aspectos também reside a contribuição teórica do estudo, uma vez que a abordagem contingencial, quando aplicada a organizações pertencentes ao setor público, gera reflexões sobre a alocação eficiente dos recursos em função de fatores contextuais. Mesmo sob condicionamento legal e normativo, o que pode tornar menos flexível a estrutura das organizações, esta pesquisa demonstrou que as variáveis contextuais interferem nas estruturas internas. Nesse sentido, deve-se questionar, em estudos posteriores, até que ponto o tamanho pode contribuir com a eficiência dos mecanismos de controle interno.

\section{CONCLUSÕES}

Além da rejeição da hipótese, considerações pertinentes são enumeradas nesta conclusão. Nas entidades públicas analisadas, o tamanho demonstrou relação com a autoavaliação da estrutura de controle interno em diferentes níveis.

Órgãos que desempenham funções contábeis diretas e indiretas têm melhor autoavaliação, o que poderia sugerir que a capacidade técnica e a especialização das funções ajudam a estrutura do controle interno, mas os resultados mais baixos estão nos aspectos referentes à estrutura do controle interno e à avaliação de riscos. Esses resultados reforçam a importância que o gestor público detém na condição de líder e reprodutor de atitudes gerenciais eficientes.

Condizente ao exposto, nos órgãos analisados, a atuação e responsabilidades da alta gestão é o fator mais relevante na avaliação do controle interno; a estrutura organizacional tem menor importância. Isso ajuda a explicar a ausência de correlação e interferência das variáveis de tamanho organizacional sobre a medida global alusiva ao controle interno dos órgãos. Contudo, o tamanho da entidade se relaciona com aspectos específicos: positivamente com plano de longo prazo, informação e qualificação; negativamente com a estrutura.

A influência negativa do tamanho organizacional sobre a avaliação da estrutura do controle interno tem implicações pertinentes, dentre as quais percebe-se que a despesa empenhada é a melhor proxy em relação ao orçamento, talvez porque trate de gastos efetivados. O capital humano também influencia o controle interno, mas o quantitativo de ativos explica melhor a variância da autoavaliação. Conjectura-se haver um gargalo em termos de estrutura econômico-financeira, capital humano e controles internos.

Destaca-se que é possível que a previsão legal possa engessar as estruturas de controle interno dos órgãos, já que a norma define as mesmas regras para todas as organizações, independentemente das suas características e da complexidade. Essa peculiaridade ajudaria a explicar os resultados alcançados no contexto da administração pública brasileira.

A pesquisa tem limitações, como não verificar outras contingências ou mesmo análise em outros contextos (corporativo privado) e outros períodos. A teoria sugere que existem diversas contingências, como a tecnologia ou a estrutura organizacional. Esta pesquisa limita-se apenas a analisar a contingência tamanho, o que não invalida os resultados. Ao contrário, possibilita emergir novas discussões junto à teoria da contingência na administração pública brasileira. A replicação da pesquisa em órgãos de outros estados e 
municípios, além da acessibilidade a um maior número de formulários de autoavaliação ajudaria a gerar outras inferências. Estudos futuros devem explorar essas limitações.

Além disso, abrem-se lacunas que serão exploradas em investigações futuras. Uma abordagem mais detalhada talvez possa ser direcionada ao custo da atividade e "setor" do controle interno dos órgãos e do pessoal alocado em área específica.

\section{REFERENNCIAS}

Alves, A. G. S., Moraes, V. F. Júnior (2016). O sistema de controle interno da gestão pública do poder executivo do Município de Patos/PB. Revista Evidenciação Contábil \& Finanças, 4(3), 56-71.

Barbieri, A. R., Hortale, V. A. (2002). Relações entre regulação e controle na reforma administrativa e suas implicações no sistema de saúde brasileiro. Revista de Administração Pública, 36(2), 181-193.

Beuren, I. M., Zonatto, V. C. S. (2014). Perfil dos artigos sobre controle interno no setor público em periódicos nacionais e internacionais. Revista de Administração Pública, 48(5), 1135-1163.

Braga, J. G. Neto, Vasconcelos, A. C., De Luca, M. M. M. (2013). Controle interno nos relatórios anuais dos estados brasileiros à Luz da NBC T 16.8. Cadernos Gestão Pública e Cidadania, 18(62), 123-142.

Bryan, D. B. (2017). Organized labor, audit quality, and internal control. Advances in Accounting, 36, 11-26.

Cannavina, V. C., Parisi, C. (2015). Gestão pública em entidades brasileiras: adequação dos procedimentos de controles internos às normas COSO/INTOSAI. Revista Universo Contábil, 11(3), 6-26.

Capovilla, R. A., Gonçalves (2018). Avaliação do ambiente de controle interno por meio de modelo de maturidade em organizações governamentais. Revista Contemporânea de Economia e Gestão, 16(2), 146-185.

Chalmers, K., Hay, D., Khlif, H. (2018). Internal control in accounting research: a review. Journal of Accounting Literature. In press, https://doi.org/10.1016/j.acclit.2018.03.002

Chang, Y-T, Chen, H., Cheng, R. K., Chi, W. (2019). The impact of internal audit attributes on the effectiveness of internal control over operations and compliance. Journal of Contemporary Accounting \& Economics, 15(1), 1-19.

Choi, J-H., Lee, J., Sonu, C. H. (2013). Determinants of human resource investment in internal controls. China Journal of Accounting Research, 6(1), 167-185.

Clinton, S. B., Pinello, A. S., \& Skaife, H. A. (2014). The implications of ineffective internal control and SOX 404 reporting for financial analysts. Journal of Accounting and Public Policy, 33, 303-327.

Collis, J., Hussey, R. (2005). Pesquisa em administração: um guia prático para alunos de graduação e pós-graduação (2a ed). Porto Alegre: Bookman.

Dallabona, L. F., Nardelli, L. T., Fernandes, A. R. V. (2019). Variáveis contingenciais e sistemas de controle gerencial predominantes em uma rede de supermercados do Brasil. Revista Evidenciação Contábil \& Finanças, 7(1), 58-77.

Dervishi, A., Kadriu, I. (2014). Decision making under the conditions of risk and uncertainty in some enterprises of Prishtina and Ferizaj. European Scientific Journal, 1 (special issue), 224-228.

Diniz, J. A., Ribeiro, J. F. Filho, Libonati, J. J., Fragoso, A. R. (2004, julho). Controle interno na administração pública municipal: aplicação da análise discriminante para modelar uma congruência com o controle externo. Anais do Seminário USP de Contabilidade e Controladoria, São Paulo, SP, Brasil, 4.

Donaldson, L. (1999). Teoria da contingência estrutural. In S. R. Clegg, C. Hardy, W. R. Nord (Org.). Handbook de estudos organizacionais (Vol. 1, Chap. 3, pp. 105-133). São Paulo: Atlas.

Doyle, J., Ge, W., McVay, S. (2007). Determinants of weaknesses in internal control over financial reporting. Journal of Accounting and Economics, 44, 193-223.

Fávero, L. P., Belfiore, P. (2017). Manual de análise de dados: estatística e modelagem multivariada com Excel', SPSS e Stata. Rio de Janeiro: Elsevier.

Henrique, J. C., Chagas, L., Guimarães, V. A., Leal, I. C., Jr. (2016). Análise da sistemática de controle interno do município de Três Rios. Revista Pensamento Contemporâneo em Administração, 10(3), 31-41. 
Hickson, D. J., Hinings, C. R., Schneck, R. E., Pennings, J. M. (1971). A strategic contingencies' theory of intraorganizational power. Administrative Science Quarterly, 16(2), 216-229.

Ji, X., Lu, W., Qu, W. (2015). Determinants and economic consequences of voluntary disclosure of internal control weaknesses in China. Journal of Contemporary Accounting \& Economics, 11, 1-17.

Kim, Y., Park, M. S. (2009). Market uncertainty and disclosure of internal control deficiencies under the SarbanesOxley Act. Journal of Accounting and Public Policy, 28, 419-445.

Lino, A. F., Carvalho, L. B., Aquino, A. C. B., Azevedo, R. R. (2019). A falta de trabalho institucional e mudanças organizacionais incompletas em municípios brasileiros. Revista de Administração Pública, 53(2), 375-391.

Linsley, P. M., Shrives, P. J. (2006). Risk reporting: a study of risk disclosures in the annual reports of UK companies. The British Accounting Review, 38, 387-404.

Liu, C., Lin, B., Shu, W. (2017). Employee quality, monitoring environment and internal control. China Journal of Accounting Research, 10, 51-70.

López, D. M., Peters, G. F. (2010). Internal control reporting differences among public and governmental auditors: the case of city and county Circular A-133 audits. Journal of Accounting and Public Policy, 29(1), 481-502.

Loureiro, M. R., Teixeira, M. A. C., Moraes, T. C. (2009). Democratização e reforma do estado: o desenvolvimento institucional dos tribunais de contas no Brasil recente. Revista de Administração Pública, 43(4), 739-772.

Lu, Y., Cao, Y. (2018). The individual characteristics of board members and internal control weakness: evidence from China. Pacific-Basin Finance Journal, 51(1), 75-94.

Maletič, M., Maletič, D., Gomišček, B. (2018). The role of contingency factors o the relationship between sustainability practices and organizational performance. Journal of Cleaner Production, 171, 423-433.

Mazulo, E. S., Vieira, P. R. C., Silva, A. C. M. (2013). Investigação acerca da relação existente entre a imagem e o desempenho percebido do controle interno no Exército Brasileiro: estudo com emprego de análise fatorial e regressão múltipla. Revista ADM.MADE, 17(1), 43-59.

Monteiro, R. P. (2015). Análise do sistema de controle interno no Brasil: objetivos, importância e barreiras para sua implantação. Revista Contemporânea de Contabilidade, 12(25), 159-188.

Oliveira, A. S., Callado, A. A. C. (2018). Fatores contingenciais e o controle gerencial: uma avaliação em organizações não governamentais (ONGs) brasileiras. Advances in Scientific and Applied Accounting, 11(1), 092-109.

Piccoli, M. R.; Balestrim, R. S.; Rover, A. (2015). O controle interno municipal sob a ótica do controle externo: estudo de caso em três municípios da região do meio oeste de Santa Catarina. Revista de Contabilidade UFBA, 9(2), $72-90$.

Queiroz, D. B., Nobre, F. C., Silva, W. V., Araújo, A. O. (2013). Transparência dos municípios do Rio Grande do Norte: avaliação da relação entre nível de disclosure, tamanho e características socioeconômicas. Revista Evidenciação Contábil \& Finanças, 1(2), 38-51.

Santos, P. M., Bernardes, M. B., Rover, A. J., Mezzaroba, O. (2013). Ranking dos tribunais de contas brasileiros: uma avaliação a partir dos padrões web em governo eletrônico. Revista de Administração Pública, 47(3), 721-744.

Shu, W., Chen, Y., Lin, B., Chen, Y. (2018). Does corporate integrity improve the quality of internal control? China Journal of Accounting Research, 11, 407-427.

Silva, F. A., Mário, P. C. (2015). O processo de formulação e monitoramento do planejamento estratégico de tribunais de contas sob a ótica da nova sociologia institucional. Revista de Administração Pública, 49(6), 1401-1427.

Silva, F. F., Celestino, E. J. M., Melo, C. L. L., Mól, A. L. R. (2017). Influência das variáveis de gestão econômicofinanceira e de internacionalização na governança corporativa das companhias listadas na BM\&FBovespa. Revista Mineira de Contabilidade, 18(3), 52-64.

Souza, M. Junior, Silva, M. Z. (2016). Gestão pública estadual: percepção dos gestores sobre a qualidade dos controles internos. Revista Catarinense da Ciência Contábil, 15(46), 47-60.

Pederneiras, M. M. M., Lopes, J. E. G., Ferreira, J. O. L., Santos, V. M., Ribeiro, J. F., Filho. (2018). Marcos regulatórios do controle interno: um estudo na ambientação com o controle externo e o controle social no Brasil. Revista de Administração e Contabilidade da Unisinos, 15(1), 2-17. 
Peterson, A. N. (2018). Differences in internal control weaknesses among varying municipal election policies. Journal of Accounting and Public Policy, 37, 191-206.

Pugh, D. S., Hickson, D. J., Hinings, C. R., Turner, C. (1969). The context of organization structures. Administrative Science Quarterly, 14(1), 91-114.

Roesch, S. A. (2006). Projetos de estágio e de pesquisa em administração: guia para estágios, trabalhos de conclusão, dissertações e estudos de caso (3a ed.). São Paulo: Atlas.

Solomon, J. F., Solomon, A., Norton, S. D., Joseph, N. L. (2000). A conceptual framework for corporate risk disclosure emerging from the agenda for corporate governance reform. British Accounting Review, 32, 447-478.

Speeden, E. A., Perez, O. C. (2020). Fatores que impactam na qualidade do planejamento orçamentário dos municípios do estado de São Paulo. Administração Pública e Gestão Social, 12(1), 1-22.

Taffarel, M., Almeida, L. B., Pereira, M. J., Clemente, A. (2018, novembro). Práticas de contabilidade gerencial e tamanho das empresas: uma análise do segmento madeireiro no estado do Paraná. Anais do Congresso Brasileiro de Custos, Vitória, ES, Brasil, 25.

Thomson, D. (2010). Exploring the role of funders' performance reporting mandates in nonprofit performance measurement. Nonprofit and Voluntary Sector Quarterly, 39(4), 611-629.

Tonelotto, D. P., Righetto, P., de Moraes, V. M., Crozatti, J. (2019). Hospitais de alta complexidade do estado de São Paulo: uma análise comparativa dos níveis de eficiência obtidos pelos modelos de gestão de administração direta e de organização social. Administração Pública e Gestão Social, 11(4), 1-22.

Valentim, I. C. D., Silva, L. O., Passos, J. G. (2016). Controle interno e gestão: uma revisão baseada em estudos brasileiros. Revista Interface, 3(1), 69-89.

Waterhouse, J. H., Tiessen, P. (1978). A contingency framework for management accounting systems research. Accounting, Organizations and Society, 3(1), 65-76.

Woods, M. (2009). A contingency theory perspective on the risk management control system within Birmingham City Council. Management Accounting Research, 20(1), 69-81. 